\title{
SURCOS SOBRE EL ASFALTO AL 9N METROPOLITANO. REFLEXIÓN DEL FENÓMENO MIGRATORIO ESPAÑOL
}

\section{SPANISH RURAL FLIGHT: NOV.9 AND THE EXPERIENCE OF THE INDEPENDENCE STRAW POLL}

\author{
José Ma Cortés Martí \\ Universidad de Barcelona. España/Spain \\ josepmcortesmarti@ub.edu
}

Recibido/Received: 29/01/2015

Modificado/Modified: 15/04/2015

Aceptado/Accepted: 29/04/2015

\section{RESUMEN}

El proceso migratorio comportó, para la sociedad de acogida, una transformación de primer orden en la estructura social y económica pero también en el imaginario colectivo. La actual población de nacionalidad española del Barcelonés norte en sus tres cuartas partes proviene de otras regiones españolas, básicamente de Andalucía. En las elecciones europeas del 2014 y en la consulta del 9N se detecta una tendencia de voto distinta al resto del territorio explicada por la variable clase social y en el imaginario de adscripción territorial.

\section{PALABRAS CLAVE}

Migración, emigración, imaginario, desigualdad, estigmatización.

\section{SUMARIO}

1. El cine como recurso analítico de la sociología. 2. El relato de cambio social en el cine. 3. Contexto migratorio 1950-1975. 4. Textualidad de la migración en España. 5. Cuarenta años después el 9N desenlace de una realidad. 6. Conclusión. Bibliografía.

\begin{abstract}
The migration process described above for society behaved host first order transformation of the social and economic structure but also in the collective imagination. The current population of Spanish nationality North Barcelones in its three quarters comes from other Spanish regions, mainly in Andalusia. In the European elections of 2014 and in the elections $9 \mathrm{~N}$ a tendency to vote differently to the rest of the territory explained by social class variable and in the imagery of territorial attachment is detected.
\end{abstract}

\section{KEYWORDS}

Migration, emigration, imagery, inequality, stigmatization.

\section{CONTENTS}

1. Movies as an analytical resource of sociology. 2. The story of social change in the movies. 3. Context migration from 1950 to 1975 . 4. Textuality of migration in Spain. 5. Forty years after the $9 \mathrm{~N}$ outcome of a reality. 6. Conclusion. References. 


\section{CINE COMO RECURSO ANALÍTICO EN LA SOCIOLOGÍA}

El gran desarrollo de las tecnologías audiovisuales nos permite incorporarlas como herramientas pedagógicas y testimonios de una época convirtiéndose en documentos sociológicos que relatan con realismo y rigurosidad procesos de vida cotidiana. Como toda producción humana el cine también participa de la dimensión sociológica, convirtiéndose algunas veces en espejo de la realidad social. Esto, sin olvidar que el cine también es un producto de mercado con lo que ello conlleva de relato social. Sin embargo, para el sociólogo el cine puede convertirse en una herramienta de análisis social siempre que se cumplan unas condiciones: que el relato refleje como un espejo la realidad social que se desea estudiar; que el correlato cinematográfico cristalice el imaginario objeto de estudio; y que en cierta medida, se debe tener presente, que el cine manifiesta la manera de pensar del momento que se produjo el film.

El cine permite a la sociología indagar como se construyen los procesos sociales en su plano más micro-sociológico, sin olvidar que la ficción del cine recrea la realidad, a la vez que la imita y algunas veces la exagera para expresar aquello que el guionista y el director desean transmitir. Seria un error caer en la ilusión de que el relato cinematográfico es la realidad social que se desea estudiar, otra cosa es la meta-realidad del fenómeno fílmico, ya que es consabido, la ficción supera la mera recreación de la realidad y en su interior caben la ilusión, la fantasía o la locura. Ahora bien, construir la ficción aunque en si misma sea un acto de realidad, la ficción recrea, es decir la vuelve a crear la realidad observada como acto imitativo, aspecto éste no menor, ya que acaece la función de espejo.

Teniendo presente estos prolegómenos de carácter heurístico y actitud metodológica, el cine español sin pretender de manera manifiesta ser cine social, se ha caracterizado por hacer de la realidad ficción haciendo una descripción del mundo que le rodea. Una descripción que por ser irreal es mi misma construye el mundo social a partir del espejo como vía de entender y comprender por un lado, en denunciar y cambiar al realidad por el otro. Por lo tanto, el cine español, a igual que buena parte del cine europeo de la segunda mitad del siglo $\mathrm{XX}$, reconocía la manera de vivir de sus ciudadanos, mediante el lenguaje fílmico como un acto de responsabilidad artística. En fin, el cine para la sociología parte de reconocerlo como la reconstrucción de significados que articulan los fenómenos sociales como sujeto del relato manifestando la descripción de un hecho social.

\section{EL RELATO DE CAMBIO SOCIAL EN EL CINE}

Un ejemplo de relato fílmico del cambio social acaecido durante la segunda mitad del siglo XX es evocado en los fotogramas de aquellas películas sociológicamente juveniles como la de José Luis Garci Solos en la madrugada "habiéndonos hecho mayores" entre Surcos y Plácido. Con maestría literaria José Antonio Nieves Conde, generación Jarama (Sánchez Ferlosio, R. 1955) y La Colmena (José Cela, C. 1951), nos describía con neorrealismo fílmico las penalidades de la familia que emigraba a la gran ciudad para "sembrar proyecto". En aquel momento, a pesar de que se conocía, los destrozos provocados a la modernidad aún no se habían repuesto. Debían, todavía transcurrir unos años, no muchos por cierto, para que el brioso moto-carro de Plácido, Berlanga con maestría pictórica, a lo Fellini, lo hiciera transitar entre calles adoquinadas y caminos polvorientos, reconstruyendo destrozos y cimentando fundamentos. 
Quisiéramos o no, la España "milagrosa" lo fue a partir del Concilio Vaticano II. Nos acercábamos, por fin, más a Boccaccio 70 que a la Dolce Vita. Sin olvidar pero, que nosotros también fuimos emigrantes, irónicamente narrado en Vente a Alemania Pepe, antes del Pacto del 78. "Abandonamos" el moto-carro de Plácido para ir con coche: El turismo un gran invento. También democratizamos el privilegio de ser universitario, en una sola generación, perfectamente reflejado en Margarita se llama mi amor. Se extendió el derecho de las vacaciones pagadas cómicamente expresado en Verano 70 Por fin, nuestra sociedad por primera vez en su historia era mayoritariamente mesocrática. Nos modernizamos; ya no éramos aquel Cateto a babor perplejo al cambio sino el Soldadito español atento a las transformaciones. Las verdes praderas allanaron el Pacto de 1978, los Pactos de la Moncloa. No obstante, Todos al suelo y Asalto en el Banco Central denuncian con ironía y dramatismo las dificultades técnicas de la que ha sido hasta ahora la actual arquitectura de la democracia española.

\section{CONTEXTO MIGRATORIO 1950-1975}

El crecimiento económico y el cambio social de los últimos cincuenta años del siglo $\mathrm{XX}$ en España es sin duda la historia de un proceso migratorio intenso en territorio nacional. El llamado Milagro de la Década Prodigiosa, los sesenta y los setenta del siglo XX, no se pueden explicar sin el transvase de efectivos demográficos del campo a las principales ciudades del territorio hispánico. Un cambio que quedó reflejado en los famosos informes socio-demográficos de la Fundación de Estudios Sociales y de Sociología Aplicada con el acrónimo, ya familiar en nuestro ámbito, de FOESSA y en los primeros programas de las sociologías urbanas y rurales de nuestras universidades así como en los manuales de estructura social de España.

Pero antes del relato mesocrático a todo color y en cinemascope de la segunda mitad de los sesenta hasta llegar a los opulentos noventa, la España de los cincuenta y de principios de los sesenta se filmó sobretodo con $35 \mathrm{~mm}$ en blanco y negro. Una población fundamentalmente labradora que deseaba locamente asimilarse al "cinemascope de Hollywood". Antes de la televisión y el automóvil, cine de barrio y moto-carro constituyeron durante los cincuentas y principios de los sesenta los anclajes del imaginario colectivo con una cierta auto-estigmatización. Dicho marco de referencia constituye la pantalla del relato que vamos analizar del proceso migratorio a partir de la imagen y el guión de una de las obras maestras del cine neo-realista español.

Surcos, película producida en 1951 dirigida por José Antonio Nieves Conde (1915-2006) director también, entre otras, de Balarrasa. Surcos sobre el asfalto, título original, a pesar de permanecer en el olvido durante mucho tiempo, hoy es considerada una de las obras maestras del neorrealismo. Estrenada el 12 de noviembre del mismo año en el cine Palacio de la Prensa de Madrid y el 26 de octubre en el cine Astoria de Barcelona. La dirección del guión y calidad en los diálogos se debe a Gonzalo Torrente Ballester (1910-1999) a partir de un argumento original de Eugenio Montes (1900-1982) político y periodista, doctorado en filosofía y letras, cuya tesis fue dirigida por el mismísimo Ortega y Gasset. A pesar que la película tenía un objetivo doctrinario para desincentivar la emigración del campo a la ciudad la realidad nos ha demostrado que a pesar de las dificultades que impuso el régimen del 39, el desplazamiento de población de aquellas zonas más deprimidas a las principales ciudades fue constante desde los inicios de los años cincuenta a mediados de los setenta del siglo XX. Sin 
duda, el fenómeno migratorio español cambió absolutamente la distribución territorial de la población concentrándose principalmente en las áreas urbanas de Madrid y Barcelona

El relato emigratorio recae en el reparto de personajes como Pepe (Francisco Arenzana), hijo mayor de la familia formada por los actores José Prada y Maria Francés (padres), Maria de Leza con el papel de Tonia (hermana pequeña) y Ricardo Lucía, con el papel de Manolo (hermano menor). Configuran la red de acogida primaria (los parientes de la ciudad): María Asquerino interpretando la "Pili" conjuntamente con Carmen Sánchez (como Engracia y madre de Pili). La red social de integración encabezada por Félix Defauze (Don Roque con el sobrenombre Chamberlain, propietario de un bar detrás del cual se esconde su actividad estraperlista) y Luis Peña (El Mellao, como personaje de rufián) conjuntamente con la panda de los tres formada por actores tan representativos de una época como Francisco Bernal en el papel de Juan y limpia botas, José M. Martín y José Villansante en los papeles de Carlos y Enrique. Como red de socialización e integración de Manolo, Manuel de Juan, titiritero de guiñol y padre de Rosario interpretado por una jovencísima Montserrat Carulla como Rosario.

Surcos como relato emigratorio define a la perfección dicho proceso y su articulación institucional: el efecto llamada como justificación del viaje, la red de acogida familiar como mecanismo de residencia primaria, la estigmatización social por la diferencia en la "mise en scene", procesos de socialización secundaria por adopción de nuevas costumbres y aprendizaje de un nuevo oficio, funcionamiento de los mercados de trabajo vía precio, vía formación, vía selección informal. Paralelamente los dos ámbitos de socialización quedan perfectamente definidos por el lugar de residencia, Lavapiés, y por las características del puesto de trabajo. Todos estos elementos constituyen un marco de referencia que describen de forma concisa el fenómeno migratorio.

\section{TEXTUALIDAD DE LA MIGRACIÓN EN ESPAÑA}

\subsection{Rito de paso: Atocha y Lavapiés}

Como primer rito de paso al "nuevo mundo" de la familia Pérez acontece en la estación de ferrocarril de Atocha bajando del tren "proveniente de Valladolid". Son recibidos por el hijo mayor, Pepe, que "hizo la mili" en Madrid y conoce bien los lares de la capital. La falta de costumbre del ajetreo capitalino se escenifica en la estigmatización directa del hijo mayor a su familia "es que son de pueblo". La escena que concreta las características, a la vez que rito de paso en el nuevo escenario vital, es en el metropolitano madrileño cuando el resto del peaje pone en evidencia la disimilitud identitaria de la familia inmigrante. El lenguaje como control social lo determinan locuciones y expresiones "paletos", "se creen que van al campo..." marcan la configuración simbólica de lo rural y lo urbano, construyendo el imaginario antitético entre campo y ciudad (con una intención absolutamente doctrinaria del régimen, aspecto que no voy extenderme por no ser el ámbito de dicho análisis, aunque como analistas de sociedad no podemos obviar el marco de referencia en el cual se construyó el discurso argumental de Surcos).

La construcción institucional de la red de acogida queda perfectamente delimitada cuando llegan al "barrio" en la estación metro de Lavapiés, todo un símbolo de referencia a partir de connotación simbólica urbana. El ritual de entrada al "nuevo mundo" no está exento de dificultades; interpelación del guardia urbano, con mirada requisitoria y sequedad en la respuesta, no suponen obstáculos para construir una vida mejor. El panorama escénico se refuerza en la llegada a la Corrala (conjunto de alojamientos humildes formando una sola 
unidad constructiva de tres o cuatro plantas, patio interior amplio y aireado, donde se encuentra la escalera principal por la cual se accede a cada una de las plantas donde se encuentran cada uno de los alojamientos siguiendo los cánones constructivos clásicos del Madrid humilde) la familia Pérez sube despacio hasta el piso de los parientes. Observados y vigilados por el vecindad son objeto de estigmatización "de dónde vendrán éstos paletos ..."

\subsection{Socialización: novación y duelo}

El espacio compartido refuerza la red familiar como agente de socialización secundaria Pili, Engracia y Pepe- dónde se manifiesta de manera más nítida el relato entre tradición (padre) y novación (hijo mayor), enfrentando lo rural a lo urbano. El proceso de substitución de los valores sociales no suele ser directo sino más bien refractario y lento pero constante. El cambio en las estructuras se expresa en la modificación de las instituciones reforzado por las expectativas de cada uno de los miembros. En la intimidad familiar del comedor se cristaliza el rito de abandono cognitivo del campo incorporándose el imaginario de la gran ciudad:

- Jo decía traerlos a todos (pollos y gallinas) para poner un gallinero (padre)

- A éste no se le va la idea del comer y lo de la huerta (madre)

- Lo decía por ganar unas pesetas (padre)

- Aquí el dinero se gana de otra manera; siendo "espabilado" y estando en todo (Engracia)

- Todos sabemos lo del nuestro oficio (padre)

- Trabajadores como nosotros habrá pocos (madre)

- Bahhh. Lo que da dinero. Vosotros no lo entendéis. Pero lo que yo os digo es que hay que ganar dinero como sea, porque aquello del pueblo con un jornal es para morirse, uno debe de sacar lo que ha aprendido en el mundo, y yo he aprendido que se gana pasta o le dan a uno de "lao" (Pepe)

No obstante la dialéctica durante todo el relato pivota en entre "estar" pero "no ser", esto quiere decir convivir sin asumir los valores, y por otro "ser" y "estar" integrase en la sociedad de acogida como acto de realización. Los padres con roles actantes -al inicio de la senectud- no actualizan los valores pero se definen dos realizaciones potenciales: por una parte la madre quiere, virtualiza el cambio, lo autoriza; por el contrario el padre no lo quiere, reforzando la narración escéptica ante cambio. En segundo plano argumental se desarrolla un conflicto de poder entre los progenitores que conllevará al enfrentamiento de género explicita por la autorización o desautorización al cambio social. En el plano fílmico la escena que refleja fielmente la aversión a todo aquello que supone novación es cuando Pepe y Pili aprehenden al resto de familia con el rosario sentados alrededor de la mesa del comedor:

- Ya os dije que estas cosas están bien "pa” el pueblo (Pepe)

- Lo hemos hecho siempre y no hay que dejarlo (Padre)

- Mirada inquisitoria de la madre implícitamente dando razón a Pepe.

La migración es una situación de cambio con el objetivo de mejorar la situación personal y familiar. Las expectativas de beneficio van acompañadas por sensaciones de duelo por el alejamiento del ámbito primario de socialización y el riesgo que conlleva en nuevo contexto. El estímulo del "efecto llamada" es mediatizado desde un primer momento por la presuposiciones del sujeto ante el cambio social: el padre que hará "56 (años) por simiente" inserto en el escenario de plaza de Lavapiés es incrédulo y lanza una invectiva "pero esto no es lo que él decía" a Pepe ya habituado al ajetreo urbano. Manolo el hijo menor presupone "si 
lo sabrá él, pasó aquí la mili". Tonia, hija menor confirma "Pues a mi gusta". La madre reconfirma "estamos aquí gracias a él". "Si no fuera por eso -por haber hecho la mili- os pudriríais todos cavando la tierra" responde Pepe. Los primeros planos nos reflejan absolutamente el escenario urbano con toda su crudeza. Independientemente de la doctrina propagandística del régimen en contra del abandono del campo para ir a la ciudad, la película es todo un alegato milimétricamente elaborado de la desigualdad y la pobreza con el relato migratorio como metáfora. La interpretación del duelo queda muy bien reflejada por la competencia del hacer de cada uno de los personajes.

\subsection{Desigualdad: Trabajo e integración}

El acceso al trabajo constituye el cauce de integración por excelencia de la población inmigrada. La fuerte segmentación de dicho mercado obliga a plantear el análisis sociológico no de un sólo mercado, como suele ser tradicional en la economía del trabajo, si no en el funcionamiento de distintos mercados de trabajo como mecanismo de reproducción de la desigualdad. Las actividades menos cualificadas son habitualmente cubiertas por mano de obra foránea y en mucho de los casos la necesidad obliga desempeñar actividades que rozan la ilegalidad. Surcos en diferentes planos escenifica a la perfección los ritos de paso para acceder al mercado laboral. Se diferencian cuatro procesos caracterizados por los personajes de Manuel (padre), Pepe (hijo mayor); Manolo (hijo menor) y Tonia (hija pequeña). La incorporación al mercado de trabajo por parte del padre y el hijo menor en un principio se realiza formalmente en la oficina de empleo en la cual se escenifica una oferta de trabajo mucho mayor a la demanda. La dificultad de encontrar trabajo y paralelamente el rechazo hacia aquellos que han llegado los últimos, constituyen las barreras de entrada en el mercado laboral no sólo por objetividad en la escasez de trabajo sino mediante la subjetividad cotidiana de la acción social. Dicha escena magistralmente descrita en el plano de la "oficina de colocación", cuando padre e hijo en la cola para inscribirse coinciden con tres solicitantes más que los increpan en la fila:

- De donde habrán salido... (personaje A)

- A lo mejor resultan de barrio de Salamanca (personaje B)

- Oye tú...de dónde habéis salido?? (personaje C)

- Venimos del campo (Manolo)

- Cállate (Padre)

- !!!Vayaiii es que le tiene usted prohibido el hablar (personaje B)

- Esta gente no viene más que a reventarnos, por si fuéramos pocos... (personaje C)

- Después de todo tú también has venido del campo (personaje A)

- Eran otros tiempos y entonces no le sacaba el pan a nadie... (personaje C)

Pepe y Tonia se incorporan al mercado mediante la red informal encabezada por el "amo" del bar "Chamberlain" en el papel de estraperlista que ofrece trabajo aparentemente legal, pero mal pagado, y más bien pagado si se asume el riesgo de la ilegalidad. Pepe, mediante la ayuda de Pilar, encuentra trabajo pero "haciéndolo más barato". Tonia, la más joven ambiciona ser artista (cupletista) una realidad mucho más luminosa y hace cualquier cosa para abandonar su actividad de chica de las faenas. Manolo, el hijo menor, a punto de caer en la marginalidad, el noviazgo con una chica sencilla hija de un guiñolista, del cual Manolo aprende el oficio, se integra en la sociedad de acogida por los dos cauces más tradicionales y positivamente sancionados. Finalmente Pepe deseando ganar más asume el riesgo del estraperlo y Tonia mediante una selección informal es rechazada del ámbito artístico y reorientada como dama de compañía del "amo". 


\section{CUARENTA AÑOS DESPUÉS: EL 9N DESENLACE DE UNA REALIDAD}

El proceso migratorio anteriormente descrito comportó para la sociedad de acogida una transformación de primer orden en la estructura social y económica pero también en el imaginario colectivo. La actual población de nacionalidad española del Barcelonés norte en sus tres cuartas partes proviene de otras regiones españolas, básicamente de Andalucía. El crecimiento de los municipios de dicha corona se multiplicó respecto a la población base de 1950: en Santa Coloma de Gramenet por 5, de 25.000 en 1950 habitantes se elevó a 125.000 en 1981, en Badalona y en Sant Adrià del Besòs se incrementó por 4 y por 3 respectivamente. Si analizamos las últimas elecciones en dicho territorio se perciben unas características muy específicas en la alternativa ideológica y del imaginario de adscripción territorial a partir de los resultados obtenidos en las últimas elecciones europeas de 2014 y de la consulta del $9 \mathrm{~N}$ respecto al proceso de independencia. El análisis nos permite redimensionar la opción ideológica respecto al sentimiento territorial en relación a los datos disponibles. Analicemos pues, el desenlace del gran cambio de nuestra sociedad y como el imaginario colectivo coexiste y convive en la sociedad de acogida partir de los resultados en dichos municipios que configuran la subcomarca del Barcelonés norte.

\subsection{Ideología y nacionalidad}

Las elecciones europeas habitualmente no son un termómetro fiable para la política más cercana y de corto recorrido, ya que los índices de abstención suelen ser mucho más elevados que en otro tipo de elecciones por singulares y distantes, pero dichas particularidades no están reñidas con la sinceridad de la opción política elegida y el ciudadano habitualmente muestra mayor desapego en el voto. Hay coincidencia que dichas elecciones suponen a veces una especie de catarsis para el electorado pues la carga simbólica del ejercicio del voto y de la abstención es mucho más espontánea y a la vez con mayor carga crítica. Estos mismos motivos, sin embargo, permiten esgrimir un escenario de tendencias políticas de más largo recorrido que probablemente orientaran el escenario político de proximidad.

La comparativa de los resultados electorales al Parlamento Europeo permiten confirmar que el PSC se mantiene como la opción más votada, en la subcomarca metropolitana norte, próximo al $30 \%$ en Santa Coloma, junto con Sant Adrià un $24 \%$ y Badalona un poco más del $20 \%$. En la Barcelona municipal PSC cede terreno quedándose con la mitad (de un $37 \%$ en el 2009 a un $15 \%$, en el 2014) resultados correlativos al conjunto de Catalunya (de $36 \%$ a 14\%). Las últimas estadísticas electorales del Ministerio del Interior demuestran como el PSC-PSOE a pesar del embate, el Barcelonés-Norte se conserva, por ahora, un voto generacionalmente fiel.

No obstante la personalización de la tendencia entre ideología y nacionalismo se observa en la segunda opción: en Santa Coloma es Iniciativa con un 14\%; Esquerra con un 17\% y $13 \%$ en Badalona y Sant Adrià respectivamente. La propensión en clave ideológica hacia la izquierda en Santa Coloma es mucho más nítida (PSC/ICV-EUiA) que en Badalona y Sant Adrià donde el voto de izquierda se define con un arco más nacionalista (PSC/ERC-NECatEPDD). En Barcelona ciudad prima una asíntota nacionalista con Esquerra Republicana en primera opción y CiU como segunda, seguido de PSC-PSOE. La lectura política de cada municipio perfila cierta disonancia en el área metropolitana. 
Ahora bien, donde se observa una amplia dispersión entre los municipios analizados es a partir de la tercera opción definiendo con claridad el imaginario nacionalista. PP es la tercera en Santa Coloma, CiU en Badalona y en Sant Adrià ICV-EUiA. Santa Coloma se desmarca del nacionalismo relegando a un cuarto puesto la coalición de Esquerra, seguido por Ciudadanos y PODEMOS. Tanto Badalona y como Santa Adrià confinan al PP en cuarta posición. Aunque CiU es la segunda opción más votada en Catalunya, es la quinta para Sant Adrià, y en Santa Coloma se apercibe como una opción marginal arrinconada a una sexta posición (tercera en 2009) a pesar de formar gobierno en el Ayuntamiento, iniciativas enfáticas, actos altilocuentes y el aumento de participación (pierde aproximadamente 100 votos).

\subsection{Nacionalidad y territorio}

La reclamación o exigencia de independencia en Catalunya respecto al estado español no es novedoso. Rememoremos un poco para adentrarnos en algo tan técnico como emotivo. El concepto de "Nació Catalana" aunque de significado muy ambiguo, lo encontramos ya en el siglo XVI siendo uno de los ejemplos más precoces en la formulación del estado-nación. Lengua y territorio son ingredientes recurrentes en todos los nacionalismos, aunque no siempre se dan, como por ejemplo en Escocia, donde los nacionalistas escoceses no acostumbran hablar en gaélico. Por otro lado estados como Irlanda o Polonia que no han tenido en su historia exigencias de estatalización tan nítidas como el caso de Catalunya. El caso catalán, visto en perspectiva, es un caso muy atípico, ya que tiene los ingredientes necesarios para ser una nación pero en cambio no se ha consolidado como estado. El catalanismo como corriente ideológica, como el resto de nacionalismos europeos, comienza a configurarse en el siglo XIX y lo largo del XX. No obstante, a diferencia de los otros, el catalanismo es diverso y plural. Desde las tendencias más tenues del regionalismo ibérico a las propuestas más independentistas para la consecución de un "Estat Català". Es obvio que este horizonte despliega infinidad de adscripciones territoriales, culturales e incluso técnicas, desde el derecho de la autodeterminación a igual que una colonia respecto a la metrópoli, yendo a la configuración de los "Països Catalans", hasta recorrer al tecnicismo táctico de la autonomía de derechas y al federalismo inverso de izquierdas, pasando por todas las tonalidades sentimentales: desde la mera simpatía folclórica a la acentuación más exacerbada del nacionalismo.

En segundo lugar, los datos comparativos nos ilustran muy bien de los dos procesos europeos. Escocia con una participación del $85 \%$ el $55 \%$ expresó que todavía desea pertenecer unido al Reino. En Catalunya con los datos dados como válidos, si el censo potencial es 6.228 .531 , ha votado un $37,02 \%$. Es decir del total del censo sólo un $29,89 \%$ han votado Si-Si aunque representa un $81 \%$ de la participación. Pero lo importante en estas votaciones es como se ha percibido la confección del proceso, quienes lo encabezaban y que se pretendía por parte de los partidos como CiU y ERC. Y por otro lado, no es de extrañar, que dicha consulta refleje la realidad sociológica de adscripción nacional de aquellos que no han votado y de los que si lo han hecho, con los dos extremos de sólo ser español o de sólo ser catalán, pasando por el rico abanico de tonalidades nacionales.

Así los datos de la participación en la consulta se cristaliza todavía más el imaginario nacional de la Cataluña metropolitana de principios del siglo XXI. En Santa Coloma de un censo a derecho a voto de 78.582 votaron en la consulta 17.797, es decir aproximadamente un $22 \%$ de los cuales un 62,27\% se abocó al SI-SI, SI-NO el 18,22\%, NO 11,06\%. Dicho de otro modo, del total del censo el SI-SI representa el 14\%. En Badalona de un censo de 154.660 votó 49.580 , es decir un 31,81\%. El SI-SI, representó el 72,51 del voto emitido el 
SI-NO 14,42 y el NO 6,75. En Sant Adrià del Besòs de un censo de 25.359 votaron 6.196 o sea un $28 \%$. SI-SI un $68,21 \%$, SI-NO, $16,27 \%$ y NO un $8,42 \%$. Si englobamos al análisis la no participación vemos que es mayoritaria $88 \%$ en Santa Coloma de Gramenet; $68 \%$ en Badalona y Sant Adrià del Besòs un $72 \%$.

\section{CONCLUSIÓN}

Si imagináramos la Historia moderna de España como una carretera cuyo horizonte se confunde y no puede dilucidarse el final, desde la instauración de la democracia en España, algunos hechos concretos han sido desencadenantes de cambios de rumbo, así, desde la carretera principal, hemos podido coger desvíos, atajos, y como no, equivocarnos y meternos por autopistas de peaje, algunas de ellas, demasiado caras. Recordando fechas que han ocasionado cambios de timón, destacan el 20N de 1975 fecha oficial de la muerte de Franco; el golpe de estado de Tejero en 1981 recordado como el 23F, 11M (M de marzo) de 2004 fecha de los atentados de Madrid por crepúsculos del islamismo, 15M (M de mayo) de 2011 fecha en que España comienza a indignarse y de mal recuerdo en Catalunya por la nefasta política policial de CiU. El 9N de 2014 también convertida en fecha para los anales históricos tanto en Catalunya como en el resto de España.

A mi modo de ver ésta es una, de las muchas variables, que nos permiten explicar el porque de tal proceso hasta llegar a una de las interpretaciones, de las muchas, de los datos de la consulta. En primer lugar mucho voluntariado, mucha articulación civil, algunas asambleas cívicas y estrategia de combate por parte de CiU y en menor medida de ERC, expresan una parte del carácter de la Catalunya actual. Si comparamos los dos procesos, el de Escocia y el catalán, no hace falta tener un ojo avizor para darse cuenta que son procesos muy distintos aunque el objetivo sea similar. Escocia es Reino Unido y una de las democracias más admiradas: conjuntamente con Inglaterra, Gales e Irlanda del Norte definidas como cuatro naciones constitutivas de dicho Reino. Por lo tanto el orgullo británico prevalece ante la emoción escocesa. Aquí pasa lo contrario: aunque España también es Reino, nación de naciones y regiones históricas, el orgullo nacional de la España Autonómica está por los suelos, no sólo en Catalunya si no en toda España. Lo que pasa en Catalunya también pasa en la Comunidad de Madrid con otros síntomas (exigencia de la III República Española) pero el mismo diagnóstico: España está en crisis porque el periodo constitucional iniciado 1978 finalizó.

Analizando el paisaje español la novedad de las últimas elecciones europeas es el aumento de votos entre formaciones que se ubican en la parte izquierda de la euro-cámara. La Izquierda Plural aporta 6 euro-diputados, Podemos 5, EPDD (Esquerra pel Dret a Dicidir) 2, LPD (Los Pueblos Deciden) 1, Primavera Europea 1. En la parte derecha UPyD (Unión Progreso y Democracia) 4; C's (Ciudadanos) 2. CEU siglas de Coalición por Europa integrado por CiU, PNV, Coalición Canaria, mantienen los 3 diputados arañando 40.000 votos pero cediendo peso de un tercer a séptimo lugar. Convergencia Democrática de Cataluña $(\mathrm{C}$ de $\mathrm{CiU})$ permanece en la Alianza de Liberales y Demócratas para Europa junto con UPyD y C's.

Si comparamos los resultados obtenidos en el 2014 y en el 2009 se observa un hundimiento de los partidos tradicionales PP y PSOE. De los 54 eurodiputados elegidos 16 corresponden al PP y 14 al PSOE respecto a los 24 y los 23 de 2009 con una perdida absoluta de votantes, cerca dos millones y medio respectivamente tanto para el PP como para el 
PSOE. Sin duda un reparto de votos que no es fácil de digerir por los grandes partidos y que probablemente señala un nuevo escenario político. Por autonomías Extremadura, Andalucía y Principado de Asturias el voto mayoritario se decanta a PSOE. Para el PP: Madrid, Castilla-la Mancha, Castilla-Leon, Galicia, Cantabria, La Rioja, Navarra, Comunitat Valenciana y Región de Murcia. CiU-PNV para País Vasco, y en Catalunya Esquerra Republicana. Sin embargo, a pesar que la abstención decrece un casi un 1\%, es la opción más común de los ciudadanos españoles.

Como el resto de España, en Catalunya los dos grandes partidos pierden anclajes: PPC disminuye un $30 \%$ y PSC-PSOE pierde cerca del 50\%, más de 350.000 votos. Con un aumento de la participación más marcada que el resto de España que representa unos 548.500 votos más respecto al 2009. El resultado dibuja una tendencia hacia el nacionalismo de izquierda. La disminución del PSC-PSOE y el incremento de participación de casi el 11\% alimenta a ERC y Necat-EPDD, a ICV-EUiA y otra formaciones más minoritarias como PODEMOS. ERC junto con NEcat-EPDD obtiene 594.149 que representa más de un 226\% de incremento respecto al 2009. ICV-EUiA aumenta en 138.799, que representa más el $115 \%$. En el otro espectro ideológico, probablemente la pérdida de votos del PPC van a CiU con un traspaso de un poco más de 100.000 votos. CiU obtiene un poco más de un $24 \%$ respecto a los 441.810 del 2009 como opción de la derecha en Catalunya.

En definitiva, el análisis en detalle de el Barcelonés norte, las elecciones europeas y la convocatoria por la independencia reafirman una clara vocación de izquierda y comparte la singularidad de ser español como de ser catalán. De ahí que lo más importante, para los ciudadanos de los municipios analizados, no es la adscripción nacional sino el modelo de sociedad que se asienta sobre el territorio, de tener un trabajo, crear riqueza y construir un proyecto de vida. Para terminar y a modo de reflexión, no se puede olvidar que en una democracia el ciudadano tiene el derecho y la obligación de votar de manera corresponsable para exigir a los políticos transparencia, aspecto éste absolutamente ausente en los gobiernos actuales, y facilitar, sin duda, el derecho a la participación.

\section{BIBLIOGRAFÍA}

BRUNET I ICART, I. (1992). La Lógica de lo social: M. Foucault-E. Durkheim. Barcelona: PPU.

BAUMAN, Z. (2006). Vida Líquida. Barcelona: Paidós.

BERGER, P. L.; LUCKMANN, T. (1975). La construcción social de la realidad. Buenos Aires: Amorrortu.

FOUCAULT. M. (1995). Castigar y Vigilar. Madrid: Siglo XXI.

UÑA JUÁREZ, O. (2004). Diccionario de sociología. Madrid: ESIC.

UÑA JUÁREZ, O.; HORMIGOS RUIZ, J. y MARTÍN CABELLO, A. (coords.) (2007). Las

dimensiones sociales de la globalización. Madrid: Paraninfo-Cengage.

Medios audiovisuales

ESCRIVÁ, V. (dir.) (1973). Lo verde empieza en los Pirineos. [Cinta cinematográfica]. Esp.

FELLINI, F. (dir.) (1969). Dolce Vita. [Cinta cinematográfica]. It.

FERNÁNDEZ, R. (dir.) (1961). Margarita se llama mi amor. [Cinta cinematográfica]. Esp.

FERNÁNDEZ, R. (dir.) (1970). Cateto a babor. [Cinta cinematográfica]. Esp.

GARCI, J. L. (dir.) (1979). Las verdes praderas. [Cinta cinematográfica]. Esp.

GARCI, J. L. (dir.) (1978). Solos en la madrugada. [Cinta cinematográfica]. Esp.

GARCÍA BERLANGA. J. L. (dir.) (1961) Plácido. [Cinta cinematográfica]. Esp.

GIMÉNEZ-RICO, A (dir.) (1988). Soldadito español. [Cinta cinematográfica]. Esp. 
LAPEIRA, S. (dir.) (1983). Asalto en el Banco Central. [Cinta cinematográfica]. Esp.

LAZAGA, P. (dir.) (1968). El turismo un gran invento. [Cinta cinematográfica]. Esp.

LAZAGA, P. (dir.) (1969). Verano 70. [Cinta cinematográfica]. Esp.

LAZAGA, P. (dir.) (1971). Vente a Alemania Pepe. [Cinta cinematográfica]. Esp.

MONICELLI, M.; FELLINI, F.; VISCONTI, L. y DE SICA, V. (dirs.) (1962). Boccaccio 70. [Cinta cinematográfica]. It.

NIEVES CONDE. J. A (dir.) (1951). Surcos. [Cinta cinematográfica]. Esp.

\section{Breve currículo:}

\section{José Ma Cortés Martí}

Economista y sociólogo. Doctor por la URV con la tesis "Discurso de la Racionalización del trabajo en Cataluña durante la era de la organización, 1900-1936". Profesor titular de la UB y presidente Fundación Hospital Espíritu Santo. Se forma en la Universidad de Barcelona y la Facultad de Teología (ICESB). Máster por la Escuela Superior de Empresas (EAE). Autor de Manual de Sociología de la Empresa; Historia de la Automoción en Cataluña y La Tecnópolis Catalana 1900-1936. Accésit periodismo profesional (1994) y X Premio "Fermín Caballero" 2011. Colabora en Gramenet 2.0. 